\title{
Comparing the Rightmost Group Seat Number for $K$ Groups of People Under Random Seating Assignment
}

\author{
Jason $\mathrm{Li}^{1}$ \\ ${ }^{1}$ Union County Academy for Information Technology, Scotch Plains, NJ
}

February 4, 2021

\begin{abstract}
Suppose there are $N$ seats labeled 1 to $N$ from left to right. There are $K$ groups of people that need to be seated. Denote the rightmost seating number of each group by $M_{1}, M_{2}, \ldots, M_{K}$. This article studies the probability that a group $k$ has the smallest value among $\left\{M_{l}, l=1, \ldots, K\right\}$ and its relationship with the group sizes $L_{1}, L_{2}, \ldots, L_{K}$. In particular, it is shown that when the groups are non-overlapping, the group with the fewest number of people tends to have the smallest rightmost value of $M_{k}$. This result can be generalized to the case of overlapping groups under some assumptions. However, the result does not generalize to all cases and we demonstrate this with counter examples.
\end{abstract}

\section{Problem Definition}

Suppose there are $N$ seats labeled 1 to $N$ from left to right. There are $K$ groups of people that need to be seated. To ensure everybody has a seat, the total number of people has to be less than $N$. Assuming the $K$ groups are non-overlapping, we denote the rightmost seating number of each group by $M_{1}, M_{2}, \ldots, M_{K}$. This article studies the probability that a group $k$ has the smallest value among $\left\{M_{l}, l=1, \ldots, K\right\}$ and its relationship with the group sizes $L_{1}, L_{2}, \ldots, L_{K}$. Later in the article, we extend the analysis to the case of overlapping groups.

\subsection{Illustrative Example}

Figure 1 gives an illustration of the problem of interest. In this case we have altogether 9 people from 3 groups to be seated in 9 seats: group 1 (black) consists of 2 individuals, group 2 (green) group consists of 3 individuals and group 3 (blue) consists of 4 individuals. Figure 1 gives an instance of a random 
seating arrangement. $M 1, M 2, M 3$ denote the index of the rightmost seat of the groups 1, 2 and 3 respectively. As observed in this instance, there is a correlation between the group size and the values of $M_{k}$ (small group sizes tend to have smaller value of $M_{k}$ ) which is intuitive under random seating arrangement.

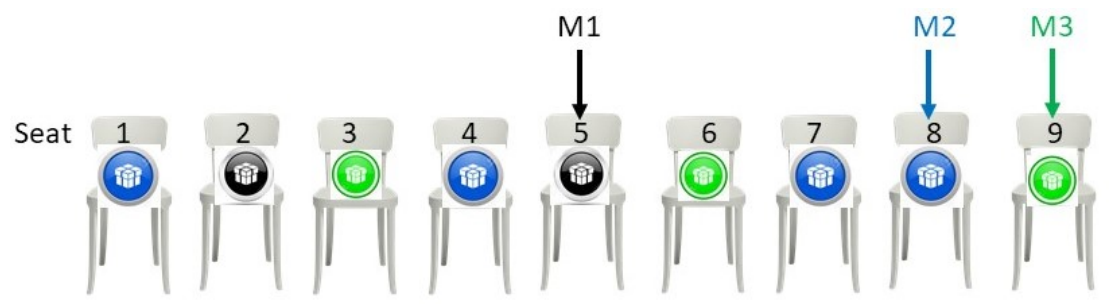

Figure 1: Illustration for Three (Colored) Groups of People with 9 Chairs. $M 1, M 2, M 3$ are the respective rightmost seats for each group, where $M 1$ is the leftmost of these three seats.

\section{Non-Overlapping Groups}

Definition 2.1. Let $S_{k}, k=1 \leq k \leq K$ be $K$ non-overlapping groups each with $L_{k}$ members respectively to be seated. For each group $k, k=1, \ldots, K$, let

$M_{k} \doteq$ seat index that are furthest to the right taken by members in group $k$.

\subsection{Comparing $M_{i}$}

Lemma 1. Let $S_{1}, S_{2}$ be two groups of people with $L_{1}$ and $L_{2}$ members respectively. If $S_{1} \cap S_{2}=\emptyset$, then the rightmost seating numbers $M_{1}$ and $M_{2}$ under random seating assignment have the property that

$$
P\left(M_{1}<M_{2}\right)=\frac{L_{2}}{L_{1}+L_{2}} .
$$

Proof. Suppose the seats are indexed by $1,2, \ldots, L_{1}+L_{2}$. It is clear that each person in the two groups has an equal probability taking the rightmost seat which is the seat indexed by $L_{1}+L_{2}$. Since everybody takes a different seat, these events are exclusive and hence the probabilities are additive. Therefore, event that $M_{1}<M_{2}$ is equivalent to the event that a member in $S_{2}$ takes the seat indexed by $L_{1}+L_{2}$, which is $L_{2} /\left(L_{1}+L_{2}\right)$ since $S_{2}$ have $L_{2}$ members.

Lemma 2. Let $S_{i}, i=1, \ldots, K$, be $K$ non-overlapping groups of people with $L_{i}$ members respectively. Let $L=\sum_{k=1}^{K} L_{k}$ be the total number of people in $K$ groups. Then for $K \geq 2$

$$
P\left(M_{k}=\max \left(M_{1}, M_{2}, \ldots, M_{K}\right)\right)=\frac{L_{k}}{L} .
$$




$$
P\left(M_{1}<M_{2}<\ldots<M_{K}\right)=\prod_{k=2}^{K} \frac{L_{k}}{N_{k}}, \text { where } N_{k}=\sum_{j=1}^{k} L_{j} .
$$

Proof. Equation (1) can be easily shown using the same argument as in the proof of Lemma 1. In this case, everybody has the same probability taking the rightmost seat of all available seats under random seating. Since group $k$ has $L_{k}$ members, the probability that one of its member taking the rightmost seat is $L_{k} / L$.

We show Equation (2) by mathematical induction. From Lemma 1, $P\left(M_{1}<\right.$ $\left.M_{2}\right)=L_{2} /\left(L_{1}+L_{2}\right)$, so Equation (2) is true when $K=2$. Now suppose this is true for $n=K$, we show that this is true for $n=K+1$.

It is necessary to show that the ordering of $M_{1}, M_{2}, \ldots, M_{K}$ is independent of the seating arrangement of group $S_{K+1}$. This is because random seating can be done sequentially by seating members in group $S_{K+1}$ first and then seating the members in the other groups. Therefore after $L_{K+1}$ seats are taken by members in $S_{K+1}$, when other groups are being seated using the remaining seats, the probability that $M_{1}<M_{2}<\ldots<M_{K}$ remains constant regardless where members in $S_{K+1}$ are seated. Therefore, the event that $M_{1}<M_{2} \ldots M_{K}<$ $M_{K+1}$ is identical to $M_{1}<M_{2}<\ldots<M_{K}$ and $M_{K+1}=\max _{k=1}^{K} M_{k}$, which are two independent events. Therefore,

$$
\begin{aligned}
& P\left(M_{1}<M_{2}<\ldots<M_{K}<M_{K+1}\right) \\
= & P\left(M_{1}<M_{2}<\ldots<M_{K}\right) P\left(M_{K+1}=\max _{1 \leq k \leq K} M_{k}\right) \\
= & \prod_{k=1}^{K} \frac{L_{k}}{N_{k}} \cdot \frac{L_{K+1}}{N_{K+1}},
\end{aligned}
$$

where the last step in the above is due to Equation (2) for $n=K$ by mathematical induction and Equation (1). This validates Equation (1) for $n=K+1$.

In the Illustrative Example 1.1, under random seating assignment,

$$
P\left(M_{1}<M_{2}<M_{3}\right)=\frac{L_{1}}{L_{1}+L_{2}} \cdot \frac{L_{3}}{L_{1}+L_{2}+L_{3}}=\frac{4}{6} \cdot \frac{3}{9}=\frac{2}{9} .
$$

\subsection{Group Index with the Smallest $M_{i}$}

For $K$ non-overlapping groups $S_{1}, S_{2}, \ldots, S_{K}$, let $Y$ be the group index such that $M_{Y}$ is the smallest of all $M_{i}, i=1, \ldots, K$. We refer $Y$ as the winning group index. From Lemma 1 we can easy obtain the following result.

Corollary 1. Let $S_{1}, S_{2}$ be two non-overlapping groups with $L_{1}$ and $L_{2}$ members respectively. Under random seating assignment,

$$
P(Y=1)=P\left(M_{1}<M_{2}\right)=\frac{L_{2}}{L_{1}+L_{2}},
$$


Furthermore if $L_{1}<L_{2}$, then $P(Y=1)>P(Y=2)$ and hence $P(Y=1)>1 / 2$ which implies that $Y$ favors the group with less members.

The following corollary extends the result for three non-overlapping groups $S_{1}, S_{2}, S_{3}$.

Lemma 3. Let $S_{1}, S_{2}, S_{3}$ be three non-overlapping groups with $L_{1}, L_{2}$ and $L_{3}$ members respectively, and let $L=L_{1}+L_{2}+L_{3}$ be the total number of members in all three groups. Under random seating assignment,

$$
P(Y=1)=P\left(M_{1}<M_{2}, M_{1}<M_{3}\right)=\frac{L_{2} L_{3}}{L}\left(\frac{1}{L_{1}+L_{2}}+\frac{1}{L_{1}+L_{3}}\right) .
$$

Furthermore,

$$
P(Y=1)>\frac{L_{2} L_{3}}{\left(L_{1}+L_{2}\right)\left(L_{1}+L_{3}\right)}=\frac{L_{2} L_{3}}{\left(L-L_{2}\right)\left(L-L_{3}\right)}
$$

If $L_{1} \leq L_{2} \leq L_{3}$, then

$$
P(Y=1) \geq P(Y=2) \geq P(Y=3),
$$

where the equality holds under the case of equal group size. In other words, Equation (5) implies the random variable $Y$ favors the group with least members.

Proof. Notice $Y=1$ implies that $M_{1}$ is the smallest among $M_{1}, M_{2}, M_{3}$. Since the groups are non-overlapping, therefore we only need to consider the following two cases: $M_{1}<M_{2}<M_{3}$ and $M_{1}<M_{3}<M_{2}$. Applying Lemma 2 to the three groups $S_{1}, S_{2}$ and $S_{3}$, we have

$$
P\left(M_{1}<M_{2}<M_{3}\right)=\frac{L_{2} L_{3}}{\left(L_{1}+L_{2}\right) L}, \quad P\left(M_{1}<M_{3}<M_{2}\right)=\frac{L_{2} L_{3}}{\left(L_{1}+L_{3}\right) L} .
$$

Therefore Equation (3) is true by summing up the above two probabilities.

We now show the bound in Equation (4). From Equation (3), we have

$$
P(Y=1)=\frac{L_{2} L_{3}\left(2 L_{1}+L_{2}+L_{3}\right)}{\left(L_{1}+L_{2}\right)\left(L_{1}+L_{3}\right)\left(L_{1}+L_{2}+L_{3}\right)}>\frac{L_{2} L_{3}}{\left(L_{1}+L_{2}\right)\left(L_{1}+L_{3}\right)} .
$$

Finally, we show $P(Y=1)$ is the largest probabilities among $P(Y=j), j=$ $1,2,3$ if $L_{1} \leq L_{2} \leq L_{3}$. For this, we only need to show $P(Y=1) \geq P(Y=2)$ if $L_{1} \leq L_{2}$ since likewise $P(Y=1) \geq P(Y=3)$ if $L_{1} \leq L_{3}$. This can be seen by directly comparing $P(Y=1)$ and $P(Y=2)$ using Equation (3). From Equation (3),

$$
\begin{aligned}
& P(Y=1)=\frac{L_{3}}{L}\left(\frac{L_{2}}{L_{1}+L_{2}}+\frac{L_{2}}{L_{1}+L_{3}}\right), \\
& P(Y=2)=\frac{L_{3}}{L}\left(\frac{L_{1}}{L_{2}+L_{1}}+\frac{L_{1}}{L_{2}+L_{3}}\right) .
\end{aligned}
$$


Comparing these two probabilities, it is easy to see that if $L_{1} \geq L_{2}$,

$$
\frac{L_{2}}{L_{1}+L_{2}} \geq \frac{L_{1}}{L_{1}+L_{2}} \text { and } \frac{L_{2}}{L_{1}+L_{3}} \geq \frac{L_{1}}{L_{2}+L_{3}} .
$$

Therefore $P(Y=1) \geq P(Y=2)$ and the equality holds only when $L_{1}=L_{2}$.

Using similar proof in the corollary above, we can further extend the results on $Y$ to an arbitrary number of $K$ non-overlapping group. We shall use recursion to accomplish the task.

Theorem 1. Let $S_{k}, k=1, \ldots, K$, be $K$ non-overlapping groups, each with $L_{i}$ members respectively, to be seated under random seating assignment. Let $L=\sum_{k=1}^{K} L_{k}$ be the total number of people in the $K$ groups and

(i) For any $k-1$ groups $S_{i_{1}}, S_{i_{2}}, \ldots, S_{i_{k-1}}$, define

$$
p_{k}\left(S_{1} ;\left\{S_{i_{1}}, \ldots, S_{i_{k-1}}\right\}\right) \doteq P\left(M_{1}<M_{i_{j}}, j=1, \ldots, k-1\right),
$$

that is, the probability that $M_{1}$ is the smallest rightmost seating number for the $k$ groups $S_{1}, S_{i_{1}}, \ldots, S_{i_{k-1}}$. Then

$$
p(Y=1) \doteq p_{K}\left(S_{1} ;\left\{S_{2}, \ldots, S_{K}\right\}\right)=\sum_{k=2}^{K} \frac{L_{k}}{L} p_{K-1}\left(S_{1} ;\left\{S_{2}, \ldots, S_{K}\right\} \backslash\left\{S_{k}\right\}\right),
$$

where this recursion can be terminated at $K=2$ with $p_{2}\left(S_{i} ; S_{j}\right)=\frac{L_{j}}{L_{i}+L_{j}}$.

(ii) If $L_{i} \leq L_{j}$, then $P(Y=i) \geq P(Y=j)$, where the strictly inequality holds when $L_{i}<L_{j}$. This implies that $Y$ favors the group with the smaller size.

(iii) For $K \geq 3$,

$$
p(Y=1)>(K-2) ! \prod_{k=2}^{K} \frac{L_{k}}{L-L_{k}} .
$$

Proof. (i) Notice $Y=1$ implies $M_{1}$ is the smallest of all $M_{k}, k=1, \ldots, K$. We can split the case of $Y=1$ into the following $K-1$ cases where in each case, $M_{k}, j=2, \ldots, K$ is the largest of $M_{1}, M_{2}, \ldots, M_{K}$. Since members in the $K$ groups are non-overlapping, these cases are exclusive and thus the probabilities are additive. From Lemma 2 Equation (1), the probability of each case, that is, $M_{k}$ is the largest among all $M_{1}, M_{2}, \ldots, M_{K}$, is $L_{k} / L$. Notice that random seating can be done sequentially by first seating members in group $S_{k}$ randomly, and then seat members in the remaining groups $S_{1}, \ldots, S_{k-}, S_{k}, \ldots, S_{K}$ randomly. Therefore given $M_{k}$ is the largest of all $M_{1}, \ldots, M_{K}, Y=1$ is identical to the event $M_{1}<M_{j}, j=2, \ldots, K, j \neq k$, which is independent of the value of $M_{k}$. This implies

$$
p(Y=1)=\sum_{k=2}^{K} P\left(M_{k} \text { is the largest }\right) P\left(M_{1}<M_{j}, j=2, \ldots, K, j \neq k\right),
$$


which leads to Equation (7) using the definition of $p_{k}$ in Equation (6).

(ii) Without loss of generality we assume $L_{1} \leq L_{2}$ and show the inequality for $i=1$ and $j=2$. First, when $L_{1}=L_{2}$, due to the symmetry in the definition $Y$ regarding to group $S_{1}$ and $S_{2}$, under random seating, it is easy to see $P(Y=1)=P\left(M_{1}<M_{2}, M_{1}<M_{3}, \ldots, M_{1}<M_{k}\right)$ and $P(Y=2)=$ $P\left(M_{2}<M_{1}, M_{2}<M_{3}, \ldots, M_{2}<M_{k}\right)$ are identical. Now we show the strict inequality when $L_{1}<L_{2}$. Let us split $S_{2}$ into two subgroups: subgroup $S_{2}^{\prime}$ with size $L_{1}$ and subgroup $S_{2}^{\prime \prime}$ with size $L_{2}-L_{1}$. Define the rightmost seat number for members in $S_{2}^{\prime}$ and $S_{2}^{\prime \prime}$ by $M_{2}^{\prime}$ and $M_{2}^{\prime \prime}$ accordingly. Since the size of subgroup $S_{2}^{\prime}$ is identical to group $S_{1}$,

$P\left(M_{1}<M_{2}^{\prime}, M_{1}<M_{3}, \ldots, M_{1}<M_{K}\right)=P\left(M_{2}^{\prime}<M_{1}, M_{2}^{\prime}<M_{3}, \ldots, M_{2}^{\prime}<M_{K}\right)$.

Furthermore, since $S_{2}^{\prime} \subset S_{2}, M_{2}^{\prime} \leq M_{2}$ and therefore $M_{1}<M_{2}^{\prime}$ implies $M_{1}<$ $M_{2}$. Hence

$$
\begin{aligned}
P(Y=1) & =P\left(M_{1}<M_{2}, M_{1}<M_{3}, \ldots, M_{1}<M_{K}\right) \\
& \geq P\left(M_{1}<M_{2}^{\prime}, M_{1}<M_{3}, \ldots, M_{1}<M_{K}\right) .
\end{aligned}
$$

Similarly, $M_{2}<M_{j}, j \neq 2$ implies $M_{2}^{\prime}<M_{j}$, and hence

$$
\begin{aligned}
P\left(M_{2}^{\prime}<M_{1}, M_{2}^{\prime}<M_{3}, \ldots, M_{2}^{\prime}<M_{K}\right) & \geq P\left(M_{2}<M_{1}, M_{2}<M_{3}, \ldots, M_{2}<M_{K}\right) \\
& =P(Y=2) .
\end{aligned}
$$

Combine all the equality and inequalities above $(9,10$ and 11), we have $P(Y=$ $1) \geq P(Y=2)$. This is a strict inequality when $L_{1}<L_{2}$ since $M_{2}$ is not always equal to $M_{2}^{\prime}$ when the set $S_{2}^{\prime \prime}$ is non-empty.

(iii). We shall use induction to show the Inequality (8). From Lemma 3 Equation (4), we have shown that the inequality is true for $K=3$. Now assume that the inequality is true for $n=K$ and we show this is true also for $n=K+1$. From Equation (7), for $n=K+1$, using the inequality assumption (Inequality 8) when $n=K$,

$$
\begin{aligned}
P(Y=1) & >(K-2) ! \sum_{k=2}^{K+1} \frac{L_{k}}{L} \prod_{j=2, j \neq k}^{K+1} \frac{L_{j}}{L-L_{k}-L_{j}} \\
& =(K-2) ! \sum_{k=2}^{K+1} \frac{L_{k}}{L} \prod_{j=2, j \neq k}^{K+1} \frac{L_{j}}{L-L_{j}} \\
& >(K-2) ! \sum_{k=2}^{K+1} \frac{L-L_{k}}{L} \prod_{j=2}^{K+1} \frac{L_{j}}{L-L_{j}} \\
& =(K-2 !) \prod_{j=2}^{K+1} \frac{L_{j}}{L-L_{j}} \cdot \sum_{k=2}^{K+1} \frac{L-L_{k}}{L} \\
& =(K-2) ! \prod_{j=2}^{K+1} \frac{L_{j}}{L-L_{j}}\left(K-\frac{1}{L} \sum_{k=2}^{K+1} L_{k}\right)>(K-1) ! \prod_{j=2}^{K+1} \frac{L_{j}}{L-L_{j}},
\end{aligned}
$$


which demonstrates the inequality for $n=K+1$.

In the Illustrative Example 1.1, under random seating assignment, the lower bound from Equation 8 for $P(Y=1)$ is $3 / 9 \cdot 4 / 9=4 / 27$. We know from the previous section that the actual probability is $2 / 9$, which is greater than $4 / 27$.

\section{Extensions to Overlapping Groups}

In this section, we extend our study to the case of possibly $K$ overlapping groups, that is, there exist members that belong to two or more groups. In the non-overlapping case, for each group, we find the the rightmost seating number for members in each of the $K$ groups and compare these numbers for the $K$ groups. However, in this case we need to modify comparisons due to possible ties from seats taken by members with non-exclusive membership.

Let us consider the case of two groups $S_{1}$ and $S_{2}$ with an intersection $S=$ $S_{1} \cap S_{2} \neq \emptyset$. When a member in $S$ takes the rightmost seat, $M_{1}=M_{2}$ which produces a tie. To resolve the tie, we only consider the seats for those individuals that have exclusive membership, that is, individuals not in $S$. In the following, we formally define a strict comparison between the seating numbers for members of two groups $S_{1}$ and $S_{2}$, and define a winning group if the group wins the comparison.

Definition 3.1. Let $S_{1}, S_{2}$ be two groups of people with $L_{1}$ and $L_{2}$ members respectively. Let the $S$ be the intersection of $S_{1} \cap S_{2}$ with size $l$, and $S_{1}^{\prime}=S_{1} \backslash S, S_{2}^{\prime}=S_{2} \backslash S$ be subsets of $S_{1}$ and $S_{2}$ respectively after removing the intersection. Denote the rightmost seating number for members in $S_{1}^{\prime}$ and $S_{2}^{\prime}$ by $\widetilde{M}_{1}$ and $\widetilde{M}_{2}$ respectively. Then we define a strict comparison operator, $\prec$, between seats taken by members in $S_{1}$ and $S_{2}$ as follows:

$$
S_{1} \prec S_{2} \doteq \widetilde{M}_{1}<\widetilde{M}_{2}
$$

Furthermore, define the winning group label $Y$ by $Y=1$ if $S_{1} \prec S_{2}$ and $Y=2$ if $S_{2} \prec S_{1}$.

Lemma 4. For two groups $S_{1}, S_{2}$ with sizes $L_{1}, L_{2}$ members and an intersection of size l, under random seating assignment,

$$
P(Y=1)=P\left(S_{1} \prec S_{2}\right)=\frac{L_{1}-l}{L_{1}+L_{2}-2 l}
$$

Furthermore when $L_{1}<L_{2}$,

$$
P(Y=1)>\frac{1}{2} .
$$

Proof. Let $S_{1}^{\prime}=S_{1}$ and $S_{2}^{\prime}=S_{2} \backslash S$. It is clear $S_{1}^{\prime}, S_{2}^{\prime}$ are non-overlapping with sizes $L_{1}-l, L_{2}-l$ respectively. Applying Lemma 1 on $S_{1}^{\prime}$ and $S_{2}^{\prime}$ gives the desired result. This in fact generalizes Lemma 1 to the case of two possible non-overlapping groups. 
In the following we generalize the comparison operator $\prec$ to an arbitrary $K$ groups and define a winning group label $Y$.

Definition 3.2. Let $S_{i}, i=1, \ldots, K$, be $K$ possibly overlapping groups of people with $L_{i}$ members respectively. For any subset of $k$ groups, $S_{i_{1}}, S_{i_{2}}, \ldots, S_{i_{k}}$, $k=2, \ldots, K$, define

$$
S_{i_{1}} \prec\left\{S_{i_{2}}, \ldots, S_{i_{k}}\right\} \text { if and only if } S_{i_{1}} \prec S_{i_{j}}, j=2, \ldots, k \text {. }
$$

Define the winning label $Y$ as follows:

$$
Y=k \text { if and only if } S_{k} \prec\left\{S_{1}, S_{2}, \ldots, S_{k-1}, S_{k+1}, \ldots, S_{K}\right\} \text {. }
$$

Lemma 5. If none of the $K$ groups $S_{1}, \ldots, S_{K}$ are identical, then for every seating arrangement, there exists a winning group with label $Y$.

Proof. For each $k, k=1, \ldots, K$, let $M_{k}$ be the rightmost seating number for members in group $k$. It is obvious that in the case of non-overlapping group, $M_{1}, \ldots, M_{K}$ are all distinct, and the winning group is the group with the smallest $M_{k}$ among all $M_{1}, \ldots, M_{K}$.

When the groups are over-lapping, we may have ties in $M_{k}, k=1, \ldots, K$. Let the smallest of these seating numbers be $M^{(1)}$ and without loss of generality, assume that the individual taking seat $M^{(1)}$ belong to groups $S_{1}, S_{2}, \ldots, S_{k_{1}}$. If $k_{1}=1$, then we have already found the winning group. If not we continue the following process.

Let $W^{(0)}=\left\{S_{1}, S_{2}, \ldots, S_{K}\right\}$ and $W^{(1)}=\left\{S_{1}, S_{2}, \ldots, S_{k_{1}}\right\}$. Then for any $S_{i} \in W^{(0)}$ and $S_{j} \in W^{(1)}$, we have $S_{i} \prec S_{j}$. Therefore, if the winning group exist, it must be an element of $W^{(1)}$. Let the seats taken by members of groups in $W^{(1)}$ be $\mathcal{L}^{(1)}$ with the largest seat number be $M^{(1)}$. Eliminating the individual taking seat $M^{(1)}$ from $\mathcal{L}^{(1)}$, for each of the $k_{1}$ group in $W^{(1)}$, we find out the rightmost seating numbers of remaining members in the group. Let the smallest of these $k_{1}$ numbers be $M^{(2)}$. It is easy to see that $M^{(2)}<M^{(1)}$, and the winning group, if it exists, must have the (new) rightmost seating number $M^{(2)}$. Let $W^{(2)}$ be the subset of $W^{(1)}$ whose (new) rightmost seating number is $M^{(2)}$. If $W^{(2)}$ consists only one group, we stop here. Otherwise, we can eliminate the individual taking seat $M^{(2)}$ and repeat this process of elimination and finding new rightmost seating numbers for the groups in $M^{(2)}$. In this way, we created a sequence of groups that are getting smaller until we only have one group left $W^{(1)} \supset W^{(2)} \supset \ldots \supset W^{(J)}$. Since none of the two groups $S_{i}, i=1, \ldots, K$ are identical, the final set of group $W^{(J)}$ with one element exists. In fact, this final group in $W^{(J)}$ must be the winning group by construction since it wins all the comparisons with other groups.

Now we study the distribution of winning group label $Y$ in the general case of $K$ possibly overlapping groups. In particular, we are interested in generalizing Lemma 4 to the case of $K$ groups. Unfortunately, for the most general case, it is not true that the winning group label $Y$ always favor the the group with the fewest number of people. 
Theorem 2. Let $S_{i}, i=1, \ldots, K$ be $K$ possibly overlapping groups each with $L_{i}$ members respectively. Assume $L_{1}<L_{2}$, then the following is not always true,

$$
p\left(S_{1} \prec\left\{S_{2}, S_{3}, \ldots, S_{K}\right\}\right) \ngtr P\left(S_{2} \prec\left\{S_{1}, S_{3}, \ldots, S_{K}\right\}\right) .
$$

or equivalently

$$
P(Y=1) \ngtr P(Y=2) .
$$

This implies that, for $K$ subsets $S_{1}, \ldots, S_{K}$ have sizes $L_{1} \leq \ldots \leq L_{K}$, the winning group label $Y$ does not always favor the smallest group.

Proof. We construct a counter-example to demonstrate this. Suppose that we have altogether 8 individuals labeled by 1 to 8 . Define three overlapping groups as follows: $S_{1}=\{1,2,3\}, S_{2}=\{1,4,5,6\}, S_{3}=\{2,3,7\}$. It is clear that $S_{1}$ has fewer members than $S_{2}$. We compute $P(Y=1)=P\left(S_{1} \prec S_{2}, S_{1} \prec S_{3}\right)$ in the following manner. First, it is easy to see that the event $S_{1} \prec S_{2}, S_{1} \prec S_{3}$ is equivalent to the fact that $\{2,3\} \prec\{4,5,6\}$ and $\{1\} \prec\{7\}$, which are two independent events. Therefore, $P(Y=1)=3 / 5 \cdot 1 / 2=3 / 10$. The calculation of $P(Y=2)$ is more complicated. Notice that

$$
P(Y=2)=P(\{4,5,6\} \prec\{2,3\} ;\{1,4,5,6\} \prec\{2,3,7\}) .
$$

Instead of direct computation, we compute the following probability

$$
\begin{aligned}
& P(\{4,5,6\} \prec\{2,3\} ;\{2,3,7\} \prec\{1,4,5,6\}) \\
= & P(\{4,5,6\} \prec\{2,3\} \prec\{1\})-P((\{4,5,6\} \prec\{2,3\} \prec\{1\} \prec\{7\}) \\
= & \frac{1}{6} \cdot \frac{2}{5}-\frac{1}{7} \cdot \frac{1}{6} \cdot \frac{2}{5}=\frac{2}{35} .
\end{aligned}
$$

Therefore, $P(Y=2)=P(\{4,5,6\} \prec\{2,3\})-P(\{4,5,6\} \prec\{2,3\} ;\{2,3,7\} \prec$ $\{1,4,5,6\})=2 / 5-2 / 35=12 / 35$. Since $P(Y=1)=3 / 10<P(Y=2)=$ $12 / 35$, this example shows the statement in Equation (13).

From the counter example in the proof above, we can intuitively interpret the reason that the winning label $Y$ may not always favor the group with the fewest members as follows. When there are a lot of overlap between two groups, there are a lot of competition between them to be the winning group and hence each of their winning probabilities is diluted (for example, $S_{1}$ and $S_{3}$ in the example). In this case, another group with more members ( $S_{2}$ for example) may become more likely to the winning group. Although we do not have the general conclusion that the winning label $Y$ favors the set with fewer members, we can still show that in some special instances this is true.

Theorem 3. Let $S_{i}, i=1, \ldots, K$ be $K$ groups with $L_{i}$ members respectively. Assume $S_{1}$ and $S_{2}$ are disjoint with the rest of $K-2$ groups $S_{3}, \ldots, S_{K}$, which implies that none of the members in $S_{1}$ or $S_{2}$ belong to the remaining groups. If $L_{1} \leq L_{2}$ then

$$
p\left(S_{1} \prec S_{2}, S_{1} \prec S_{3}, \ldots, S_{1} \prec S_{K}\right) \geq P\left(S_{2} \prec S_{1}, S_{2} \prec S_{3}, \ldots, S_{2} \prec S_{K}\right),
$$


or equivalently

$$
P(Y=1) \geq P(Y=2),
$$

where the equality holds if and only if $L_{1}=L_{2}$. This implies that, the winning group label $Y$ favor the group $S_{1}$ with the smaller size.

Proof. We first assume $S_{1}$ and $S_{2}$ are non-overlapping. Let $S_{2}^{\prime}$ be a subset of $S_{2}$ such that the size of $S_{2}^{\prime}$ is $L_{1}$. Define $S_{2}^{\prime \prime}=S_{2} \backslash S_{2}^{\prime}$. It is easy to see by the definition of $S_{2}^{\prime}$ that

$$
p\left(S_{1} \prec S_{2}^{\prime}, S_{1} \prec S_{3}, \ldots, S_{1} \prec S_{K}\right)=P\left(S_{2}^{\prime} \prec S_{1}, S_{2}^{\prime} \prec S_{3}, \ldots, S_{2}^{\prime} \prec S_{K}\right) \text {. }
$$

Since $S_{2}^{\prime} \subset S_{2}$, it is obvious that $S_{2}^{\prime} \prec S_{2}$. Therefore,

$$
P\left(S_{2}^{\prime} \prec S_{1}, S_{2}^{\prime} \prec S_{3}, \ldots, S_{2}^{\prime} \prec S_{K}\right) \geq P\left(S_{2} \prec S_{1}, S_{2} \prec S_{3}, \ldots, S_{2} \prec S_{K}\right),
$$

and

$$
p\left(S_{1} \prec S_{2}, S_{1} \prec S_{3}, \ldots, S_{1} \prec S_{K}\right) \geq p\left(S_{1} \prec S_{2}^{\prime}, S_{1} \prec S_{3}, \ldots, S_{1} \prec S_{K}\right)
$$

Combining the above two inequalities, the result now follows. The equality is only hold when the rightmost seat for members in $S_{2}$ and $S_{2}^{\prime}$ are always identical which implies $L_{1}=L_{2}$.

\section{Conclusion}

In this article, we randomly arrange $K$ groups of people and compare the values of $M_{k}$ among each other. We show that when the groups are non-overlapping, the group with the fewest number of people tends to have the smallest rightmost

value of $M_{k}$. We also consider the generalization of this result to the case of non-overlapping groups. 